\title{
Effects of multidisciplinary team on the outcomes of colorectal cancer patients with liver metastases
}

\author{
Xingchen $\mathrm{Li}^{\#}$, Qichen Chen", Xinyu Bi, Jianjun Zhao, Zhiyu Li, Jianguo Zhou, Zhen Huang, \\ Yefan Zhang, Rui Mao, Hong Zhao, Jianqiang Cai \\ Department of Hepatobiliary Surgery, National Cancer Center/National Clinical Research Center for Cancer/Cancer Hospital, Chinese Academy of \\ Medical Sciences and Peking Union Medical College, Beijing, China \\ Contributions: (I) Conception and design: J Cai, H Zhao; (II) Administrative support: H Zhao, J Cai; (III) Provision of study materials or patients: Y \\ Zhang, Z Huang; (IV) Collection and assembly of data: X Li, Q Chen; (V) Data analysis and interpretation: X Li, Q Chen; (VI) Manuscript writing: \\ All authors; (VII) Final approval of manuscript: All authors. \\ \#These authors contributed equally to this work. \\ Correspondence to: Jianqiang Cai; Hong Zhao. Department of Hepatobiliary Surgery, National Cancer Center/National Clinical Research Center for \\ Cancer/Cancer Hospital, Chinese Academy of Medical Sciences and Peking Union Medical College, No. 17 Nanli, Panjiayuan, Chaoyang District, \\ Beijing 100021, China. Email: caijianqiang@cicams.ac.cn; zhaohong@cicams.ac.cn.
}

Background: To evaluate the effect of a multidisciplinary team (MDT) treatment modality on outcomes of patients with colorectal cancer with liver metastases (CRLMs) in China.

Methods: We retrospectively identified 236 (MDT: 46, non-MDT: 190) patients who underwent liver resection or simultaneous resection for primary colorectal cancer and liver metastases with a curative intent for CRLMs at the National Cancer Center between January 2014 and June 2018. A 1:2 propensity score matching (PSM) analysis was used to adjust for differences in baseline characteristics between the MDT group and the non-MDT group. After the 1:2 PSM analysis, 46 patients were assigned to the MDT group, and 83 patients were assigned to the non-MDT group. All statistical analyses were performed using SPSS Statistics, version 22 (Armonk, NY, USA). Statistical significance was set at a 2 -sided $\mathrm{P}<0.05$.

Results: Before PSM analysis, compared to non-MDT patients, MDT patients had worse biological characteristics and were more likely to have node-positive primary tumors ( $80.4 \%$ vs. $63.2 \%)$, multiple liver metastases $(73.9 \%$ vs. $50.5 \%)$, and liver metastases with a bilobar distribution $(73.9 \%$ vs. $50.5 \%)$. However, MDT patients had a tendency towards better overall survival (OS) ( $\mathrm{P}=0.169$, median OS: 49.7 vs. 35.2 months). After PSM analysis, no differences in clinicopathologic parameters were identified between the MDT group and the non-MDT group. Compared to non-MDT patients, MDT patients had a tendency towards better OS ( $\mathrm{P}=0.063$, median OS: 49.7 vs. 34.1 months). Multivariate analysis showed that having an MDT (HR, 0.550, 95\% CI: 0.309-0.977, P=0.041) was an independent predictor of better OS.

Conclusions: The MDT treatment modality can significantly improve the outcomes of CRLMs patients with poor biological characteristics in China.

Keywords: Multidisciplinary team (MDT); colorectal cancer with liver metastases (CRLMs); propensity score matched; outcomes

Submitted Jan 12, 2020. Accepted for publication Jul 31, 2020.

doi: $10.21037 /$ apm-20-193

View this article at: http://dx.doi.org/10.21037/apm-20-193 


\section{Introduction}

The incidence of colorectal cancer increases each year, ranking third of all malignant tumors. Colorectal cancer is the second leading cause of cancer death (1). The liver is the most common target organ for colorectal cancer metastasis, which is a significant cause of death (2). Approximately $15-25 \%$ of patients with colorectal cancer have liver metastasis at the initial diagnosis, and nearly $25 \%$ of colorectal cancer patients still have heterogeneous liver metastasis after primary tumor resection (3). The median overall survival (OS) time of patients without treatment after liver metastasis is only 6.9 months (4). Although chemotherapy can prolong the survival time of patients with liver metastasis from colorectal cancer, if the liver metastases cannot be removed, the 5-year survival rate of patients is close to zero (5). However, if R0 resection of primary tumors and liver metastases can be achieved, the 5-year survival rate can be as high as 30-57\% (6-9). Five-year OS of patients with simultaneous resection of primary tumors and liver metastases in our center is $47.8 \%$. One study showed that some patients with initial liver metastases that could not be eradicated could undergo resection or achieve no evidence of disease (NED) after neoadjuvant chemotherapy (NAC) (4). Improving the R0 resection rate and converting unresectable liver metastasis into resectable liver metastasis are key for improving the therapeutic effect for patients with colorectal cancer with liver metastases (CRLMs) and are the focus of clinical research at present.

Therefore, the multidisciplinary team (MDT) for patients with CRLMs should conduct a comprehensive personalized assessment to make treatment decisions, develop an appropriate plan, prevent the occurrence of CRLMs and improve the R0 resection rate and 5-year survival rate $(10,11)$. MDT has become increasingly popular forms of diagnosis and treatment in clinical work in recent years. The core of the MDT is a patient-centered focus, and multiple experts, including those from surgery, oncology, radiology, pathology and other related disciplines, hold discussions to define the most accurate diagnostic stage for patients and to develop the most appropriate treatment plan. Several studies have shown that patients with CRLMs have an improved prognosis with an MDT diagnosis and treatment model, and many studies have shown that having an MDT is an independent predictor of a good prognosis in colorectal cancer patients $(12,13)$. Therefore, international guidelines (including 2019NCCN, 2019CSCO and 2016ESMO) and expert consensus all recommend the diagnosis and treatment of CRLMs with the MDT treatment model. An MDT can not only clarify the diagnosis and treatment plan of patients and improve their prognosis but also promote communication and discussion among different disciplines, enhance the academic atmosphere of the hospital, promote the development and enrollment of clinical trials, and improve the professional level of young doctors.

In recent years, MDT have been established in an increasing number of medical centers, there are a large number of CRLM patients in China, and the implementation of standardized MDT modality is conducive to the diagnosis and treatment of CRLM patients, but the implementation of MDT in some non-specialized hospitals is still not standardized, furthermore, few studies have been conducted in patients with CRLMs treated with surgery. There is still a lack of high-quality research to explore the advantages of MDT modality in CRLM patients in China. In addition, medical centers are more inclined to include advanced or intractable cases in MDT, which leads to differences in baseline clinicopathological features between the MDT group and the non-MDT group, and the baseline data of these included patients have not been strictly matched and analyzed, with inconsistent intervention measures and differences in tumor stage, metastatic tumor number, CRS score, etc.; thus, the conclusions are controversial. In this study, we compared the prognosis of patients with CRLMs during the same period with or without MDT involvement through propensity score matching (PSM) analysis. We present the following article in accordance with the STROBE reporting checklist (available at http://dx.doi. org/10.21037/apm-20-193).

\section{Methods}

\section{Patients and treatment}

The study was conducted in accordance with the Declaration of Helsinki (as revised in 2013). The study (ID: NCC2019C-016) was approved by the Ethics Committee of the Cancer Hospital, Chinese Academy of Medical Sciencesethics board of, NCC2019C-016 and informed consent was taken from all the patients.

After receiving ethical approval from the Institutional Review Board, we conducted a retrospective analysis of 236 consecutive cases of pathologically diagnosed CRLM patients receiving their first liver resection at our hospital between January 2014 and June 2018. Patients with $(n=46)$ 
and without ( $\mathrm{n}=190)$ MDT involvement were selected. Patients in the two groups were matched at a ratio of $1: 2$ through PSM, and a total of 129 patients were included in the study: 46 patients with an MDT and 83 patients without an MDT. After PSM, no significant differences in baseline parameters were identified between the patients in the two groups $(\mathrm{P}>0.05)$. Synchronous CRLM was defined as the detection of liver metastasis during the diagnosis of the primary tumor or within the first 6 months. CRLM patients with high clinical risk-scoring system scores or initially unresectable liver metastases were recommended to receive NAC $(14,15)$. The NAC regimens consisted of 5 -fluorouracil/capecitabine and oxaliplatin or irinotecan. Liver resection was defined as major or minor resection. Resection of one segment was defined as minor liver resection. Surgical resection included open and laparoscopic approaches. The severity of postoperative complications was described according to the Clavien-Dindo classification system, and major complications were classified as ClavienDindo III-V (16). Patients received adjuvant chemotherapy according to the histological stage and the resection margin status. After recurrence, patients received chemotherapy, TACE or surgery.

The MDT consisted of faculty from medical oncology and surgical oncology as well as radiologists and pathologists. The treatment guidelines for CRLM in our hospital were established after group discussion and modification of the guidelines according to the latest version of the MDT guidelines. The treatment protocols for patients with CRLM after MDT discussion and any deviation from the protocol were recorded. For patients with an MDT, the chemotherapy regimen, chemotherapy cycle, timing of the operation, operative approach and treatment strategies after recurrence were all determined by the MDT.

\section{Outcome and statistical analysis}

The endpoint of interest was OS, which was defined as the interval from the date of liver resection to the date of death or the last follow-up. Categorical clinical variables were compared using the chi-square test or Fisher's exact test, and numerical variables were examined by the MannWhitney U test. Survival was compared by a log-rank test and calculated with the Kaplan-Meier method. Independent variables with $\mathrm{P}<0.10$ on univariate analysis were included in multivariate models. Forward logistic regression in the multivariate analysis was used to investigate independent factors of survival. A 1:2 PSM analysis was used to adjust for differences in baseline characteristics between the MDT group and the non-MDT group. All statistical analyses were performed using SPSS Statistics, version 22 (Armonk, NY, USA). Statistical significance was set at a 2 -sided $\mathrm{P}<0.05$.

\section{Results}

\section{Clinicopathologic Characteristics}

Among the 236 patients, most were male, and the median age was 58 years (IQR 51-64). The proportion of primary tumors located in the rectum was $42.8 \%$. One hundred eighty-two patients $(77.1 \%)$ had synchronous CRLM. The median diameter of the largest lesion was 2.5 (IQR 1.7-4.0) cm, and $44.1 \%$ of the patients had a lesion larger than $3 \mathrm{~cm}$. Of these patients, $55.1 \%$ had more than one liver metastasis, with a median of 2 lesions (IQR 1.0-4.0). The T3-T4 stage was observed in $91.9 \%$ of the patients. A node-positive primary tumor was observed in $66.5 \%$ of the patients. Ninety-four patients $(39.8 \%)$ received NAC. Adjuvant chemotherapy was received by $142(60.2 \%)$ patients. Liver metastases with a bilobar distribution were observed in 93 (39.4\%) patients. Seventy-seven patients $(32.6 \%)$ received major liver resection, and 165 patients (69.9\%) underwent synchronous CRLM resection. Forty-six patients had an MDT throughout the whole therapy process. The median number of MDTs was 2 (IQR 1-3). Among the patients, $43.6 \%$ (103/236) had postoperative complications.

No differences in the age at surgery, gender, comorbidities, preoperative carcinoembryonic antigen (CEA), metastasis diameter, tumor differentiation and primary tumor $\mathrm{T}$ stage were identified between the MDT group and the nonMDT group. Conversely, compared to non-MDT patients, MDT patients have worse biological characteristics and were more likely to be treated with NAC $(58.7 \% v s$. $35.3 \%)$ and to have node-positive primary tumors $(80.4 \%$ vs. $63.2 \%)$, multiple liver metastases $(73.9 \%$ vs. $50.5 \%)$, and liver metastases with a bilobar distribution $(73.9 \% \mathrm{vs}$. $50.5 \%)$. After 1:2 PSM, 46 patients were assigned to the MDT group, and 83 patients were assigned to the non-MDT group. No differences in clinicopathologic parameters were detected between the MDT group and the non-MDT group. The detailed clinicopathologic parameters of the two groups of patients are compared in Table 1 .

\section{Impact of the MDT on survival before PSM}

The median follow-up was 32 months. At the 
Table 1 Patient and tumor characteristics

\begin{tabular}{|c|c|c|c|c|c|c|c|c|}
\hline Item & \multicolumn{4}{|c|}{ Before $1: 2$ propensity matching } & \multicolumn{4}{|c|}{ After $1: 2$ propensity matching } \\
\hline Male, $\mathrm{n}(\%)$ & $112(58.9)$ & $32(69.6)$ & 0.185 & $144(48.3)$ & $54(65.1)$ & $32(69.6)$ & 0.603 & $86(66.7)$ \\
\hline Right hemicolon, $\mathrm{n}(\%)$ & $37(19.5)$ & $8(17.4)$ & 0.747 & $45(17.1)$ & $14(16.9)$ & $8(17.4)$ & 0.940 & $22(17.1)$ \\
\hline $\mathrm{R} 0$ resection, $\mathrm{n}(\%)$ & $123(64.7)$ & $25(54.3)$ & 0.191 & $148(62.7)$ & $45(54.2)$ & $25(54.3)$ & 0.989 & $70(54.3)$ \\
\hline Major liver resection, $\mathrm{n}(\%)$ & $64(33.7)$ & $13(28.3)$ & 0.481 & $77(32.6)$ & $31(37.3)$ & $13(28.3)$ & 0.297 & $44(34.1)$ \\
\hline Diameter of metastases $>3 \mathrm{~cm}, \mathrm{n}(\%)$ & $85(44.7)$ & $19(41.3)$ & 0.674 & $104(44.1)$ & $42(50.6)$ & $19(41.3)$ & 0.311 & $61(47.3)$ \\
\hline Multiple metastases, n (\%) & $96(50.5)$ & $34(73.9)$ & 0.004 & $130(55.1)$ & $61(73.5)$ & $34(73.9)$ & 0.959 & $94(72.9)$ \\
\hline Poor differentiation, n (\%) & $52(27.4)$ & $15(32.6)$ & 0.479 & $67(28.4)$ & $25(30.1)$ & $15(32.6)$ & 0.770 & $40(31.0)$ \\
\hline pT3-T4, n (\%) & $176(92.6)$ & $41(89.1)$ & 0.434 & 217 (91.9) & $77(92.8)$ & $41(89.1)$ & 0.478 & $118(91.5)$ \\
\hline Node-positive primary tumor, n (\%) & $120(63.2)$ & $37(80.4)$ & 0.026 & $157(66.5)$ & $65(78.3)$ & $37(80.4)$ & 0.777 & $102(79.1)$ \\
\hline Complications, n (\%) & $81(42.6)$ & $22(47.8)$ & 0.524 & $103(43.6)$ & $39(47.0)$ & $22(47.8)$ & 0.927 & $61(47.3)$ \\
\hline Neoadjuvant chemotherapy, n (\%) & $67(35.3)$ & $27(58.7)$ & 0.004 & $94(39.8)$ & $43(51.8)$ & $27(58.7)$ & 0.452 & $70(54.3)$ \\
\hline
\end{tabular}

time of analysis, 86 patients $(36.4 \%)$ had died, and 181 patients $(76.7 \%)$ had experienced disease recurrence. The median OS was 41.9 (95\% CI: 32.6-51.2) months, and the median progression-free survival (PFS) was 10.0 (95\% CI: 8.4-11.6) months. The 1 - and 3 -year survival rates were $94.4 \%$ and $53.5 \%$, respectively. The 1 - and 3 -year PFS rates were $40.1 \%$ and $19.8 \%$, respectively. Compared to non-MDT patients, MDT patients had a tendency towards better OS $(\mathrm{P}=0.169$, median OS: 49.7 vs. 35.2 months) (Figure 1). Univariate analysis revealed that non-R0 resection, complications, multiple liver metastases and neoadjuvant chemotherapy were associated with decreased OS. Multivariate analysis revealed that $\mathrm{R} 0$ resection, complications and adjuvant chemotherapy were independent predictors of OS (Table 2). However, multivariate analysis revealed that having an MDT was not an independent predictor of OS.

\section{Impact of the MDT on survival after PSM}

A 1:2 PSM analysis was used to adjust for differences in baseline characteristics between the MDT group and the non-MDT group. The median OS and the median PFS were 41 (95\% CI: 33.1-48.9) months and 7.2 (95\% CI: 5.0-9.4) months, respectively. The 1- and 3-year survival rates were $94.6 \%$ and $52.2 \%$. The $1-$ and 3 -year PFS rates were $34.9 \%$ and $16.9 \%$, respectively. Compared to nonMDT patients, MDT patients had a tendency towards better OS ( $\mathrm{P}=0.063$, median OS: 49.7 vs. 34.1 months) (Figure 2). Multivariate analysis showed that having an MDT (HR, 0.550, 95\% CI: $0.309-0.977, \mathrm{P}=0.041$ ) was an independent predictor of better OS (Table 2).

\section{Discussion}

This study is the first to confirm that having an MDT 


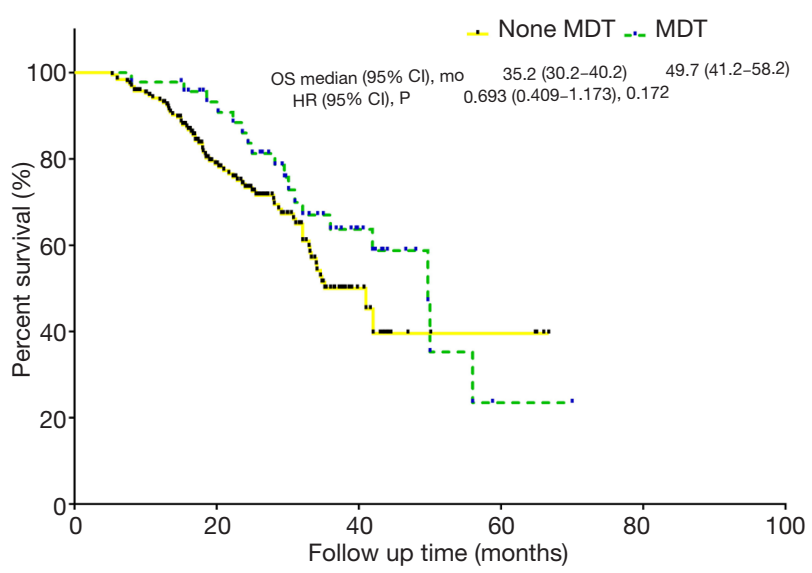

Figure 1 OS analysis of non-MDT versus MDT patients before propensity matching. OS, overall survival; MDT, multidisciplinary team.

as an independent prognostic factor for patients with CRLMs undergoing surgical treatment after PSM. In addition, multivariate analysis showed that $\mathrm{R} 0$ resection and postoperative chemotherapy were also independent factors affecting the prognosis. Compared to non-MDT patients, MDT patients were more likely to be treated with NAC (58.7\% vs. $35.3 \%)$ and to have lymph node-positive primary tumors $(80.4 \%$ vs. $63.2 \%)$, multiple liver metastases (73.9\% vs. $50.5 \%)$, and liver metastases with a bilobar distribution (73.9\% vs. 50.5\%). Patients matched for MDTs had a relatively advanced disease period, but no difference in OS was found $(\mathrm{P}=0.172)$. After PSM, having an MDT was an independent prognostic factor for postoperative OS [P=0.041; HR (95\% CI): 0.550 (0.309-0.977)]. We demonstrated the importance of MDT in the prognosis of patients with CRLMs from different perspectives. The contribution of a MDT includes increased application of NAC, advanced stage of patients received more standard comprehensive treatment, a larger proportion of patients received NAC through MDT, most of which were advanced stage and benefited significantly from NAC. In addition, careful selection of patients for appropriate treatment is important but complex. The patient's performance status, the stage of the primary tumor, the extent of the metastatic disease and the surgical or anesthesia risk of the patient should all be carefully considered. All of these factors rely on full discussion and coordination of different specialists who are involved in colorectal cancer treatment. As a result, patients can benefit from comprehensive treatment.

In recent years, the advancement of neoadjuvant therapy has substantially increased the likelihood of R0 surgery for CRLM patients, increased the volume of the remaining liver after surgery, and improved the survival rate $(17,18)$. Nearly $80 \%$ of CRLMs in patients are unresectable at initial diagnosis, and $12.5 \%$ of CRLMs are resectable through conversion therapy (19). However, the 5-year survival rate of these patients is similar to that of patients with resectable disease at the initial diagnosis (20). Therefore, under the premise of effective treatment, both conversion therapy and neoadjuvant therapy seem to improve the overall prognosis of CRLM patients. Additionally, in this study, patients with MDT involvement were more likely to be treated with NAC (58.7\% vs. $35.3 \%$ ), which may be one of the factors resulting in patients treated for CRLMs having a relatively good prognosis after MDT collaboration.

Before the 1990s, surgical treatment was the primary choice for colorectal cancer, and then a variety of comprehensive treatment methods and concepts were applied in the clinical treatment of colorectal cancer. After entering the 21 st century, MDT was gradually established in major medical centers, and their advantages have steadily emerged. Since 1998, our hospital has had an established multidisciplinary comprehensive outpatient service. In 2008, an MDT for digestive tract tumors was formally established, and case discussions were regularly organized at least once a week to facilitate preliminary screening, examination and treatment follow-up by the MDT. MDT can also reduce the number of imperfect decisions made by individual physicians (21). In addition, the important functions of and outcomes with MDT include (I) highly accurate disease staging (22); (II) limited confusion and delays in treatment; (III) highly personalized evaluation systems and treatments; (IV) good treatment cohesion; (V) high quality of life (23); and (VI) optimal clinical and survival benefits $(13,14)$.

MDT have demonstrated advantages not only in the field of digestive tract tumors but also in various fields of oncology (24). However, no strict matching analysis was conducted for the patient data included in previous studies; thus, the results may have some heterogeneity. Although PSM was used in this study to match patients' baseline data, there certain limitations remained. (I) Differences in neoadjuvant/adjuvant chemotherapy regimens were not included among the prognostic indicators, and differences in efficacy evaluations between these chemotherapy regimens and patients may lead to heterogeneity in the conclusions. (II) Not all CRLM patients were assigned to an MDT at the initial diagnosis. Some patients chose an MDT only when the initial diagnosis was difficult or when they 


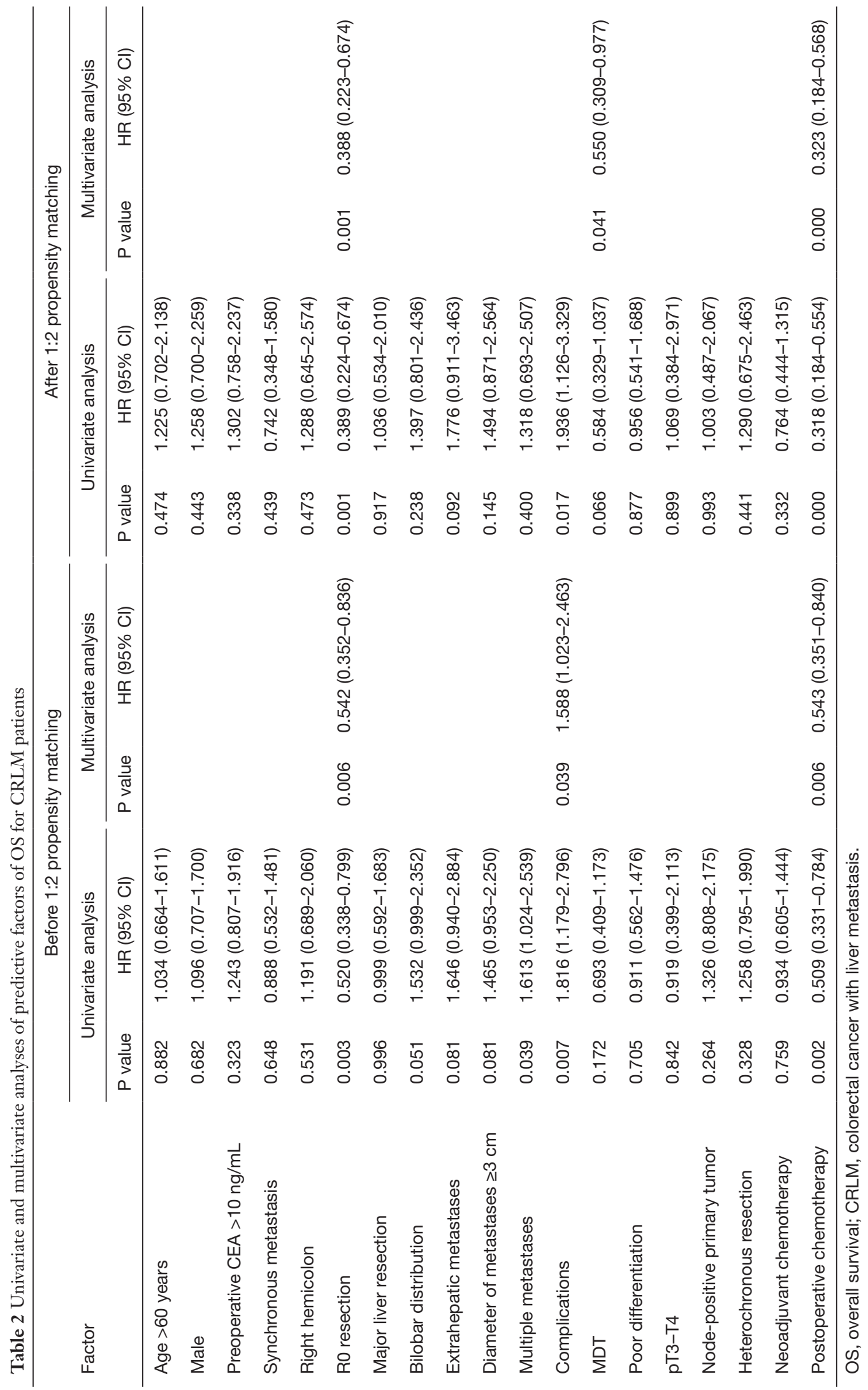




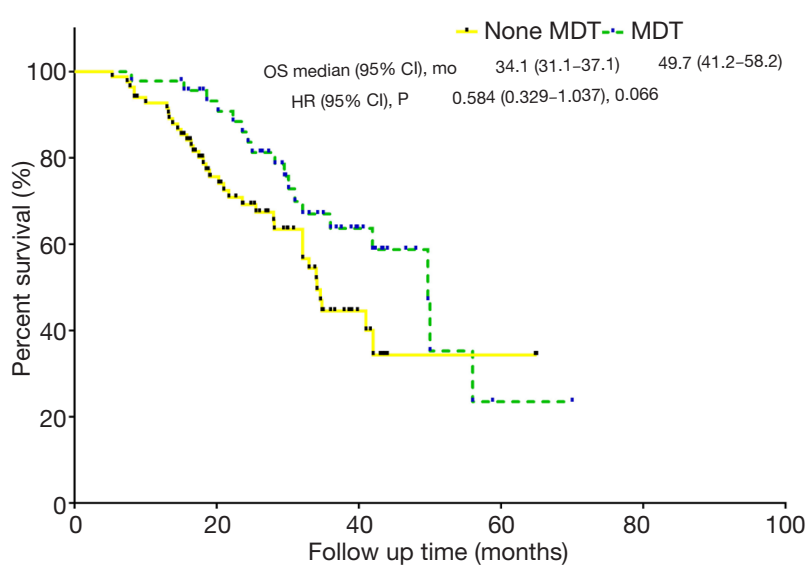

Figure 2 OS analysis of non-MDT versus MDT patients after propensity matching. OS, overall survival; MDT, multidisciplinary team.

changed their treatment plan, and a clear inclusion criterion was not determined. (III) This study was a retrospective study with low evidence for evidence-based medicine.

\section{Conclusions}

Overall, MDT demonstrated benefits for survival in patients with CRLMs. The importance of MDT in comprehensive treatment models should be scientifically recognized for CRLM patients.

\section{Acknowledgments}

We gratefully acknowledge all staff members of Department of Hepatobiliary Surgery, National Cancer Center/ National Clinical Research Center for Cancer/Cancer Hospital, Chinese Academy of Medical Sciences and Peking Union Medical College, Beijing, China, for their help and cooperation.

Funding: This work was supported by the National Capital Health Research and Development of Special (No. 20181-4021) and the National Science and Technology Major Project (No. 2018ZX10723204).

\section{Footnote}

Reporting Checklist: The authors have completed the STROBE reporting checklist. Available at http://dx.doi. org/10.21037/apm-20-193
Data Sharing Statement: Available at http://dx.doi. org/10.21037/apm-20-193

Peer Review File: Available at http://dx.doi.org/10.21037/ apm-20-193

Conflicts of Interest: All authors have completed the ICMJE uniform disclosure form (available at http://dx.doi. org/10.21037/apm-20-193). The authors have no conflicts of interest to declare.

Ethical Statement: The authors are accountable for all aspects of the work in ensuring that questions related to the accuracy or integrity of any part of the work are appropriately investigated and resolved. The study was conducted in accordance with the Declaration of Helsinki (as revised in 2013). The study (ID: NCC2019C-016) was approved by the Ethics Committee of the Cancer Hospital, Chinese Academy of Medical Sciences, and informed consent was taken from all the patients.

Open Access Statement: This is an Open Access article distributed in accordance with the Creative Commons Attribution-NonCommercial-NoDerivs 4.0 International License (CC BY-NC-ND 4.0), which permits the noncommercial replication and distribution of the article with the strict proviso that no changes or edits are made and the original work is properly cited (including links to both the formal publication through the relevant DOI and the license). See: https://creativecommons.org/licenses/by-nc-nd/4.0/.

\section{References}

1. Bray F, Ferlay J, Soerjomataram I, et al. Global cancer statistics 2018: GLOBOCAN estimates of incidence and mortality worldwide for 36 cancers in 185 countries. CA Cancer J Clin 2018;68:394-424.

2. Chan AC, Poon RT, Cheung TT, et al. Laparoscopic versus open liver resection for elderly patients with malignant liver tumors: a single-center experience. J Gastroenterol Hepatol 2014;29:1279-83.

3. Cheung TT, Poon RT, Yuen WK, et al. Outcome of laparoscopic versus open hepatectomy for colorectal liver metastases. ANZ J Surg 2013;83:847-52.

4. Sharma S, Camci C, Jabbour N. Management of hepatic metastasis from colorectal cancers: an update. J Hepatobiliary Pancreat Surg 2008;15:570-80. 
5. Van Cutsem E, Nordlinger B, Adam R, et al. Towards a pan-European consensus on the treatment of patients with colorectal liver metastases. Eur J Cancer 2006;42:2212-21.

6. de Jong MC, Pulitano C, Ribero D, et al. Rates and patterns of recurrence following curative intent surgery for colorectal liver metastasis: an international multiinstitutional analysis of 1669 patients. Ann Surg 2009;250:440-8.

7. Giuliante F, Ardito F, Vellone M, et al. Role of the surgeon as a variable in long-term survival after liver resection for colorectal metastases. J Surg Oncol 2009;100:538-45.

8. Norén A, Sandstrom P, Gunnarsdottir K, et al. Identification of Inequalities in the Selection of Liver Surgery for Colorectal Liver Metastases in Sweden. Scand J Surg 2018;107:294-301.

9. Yang AD, Brouquet A, Vauthey JN. Extending limits of resection for metastatic colorectal cancer: risk benefit ratio. J Surg Oncol 2010;102:996-1001.

10. Fahy BN, D'Angelica M, DeMatteo RP, et al. Synchronous hepatic metastases from colon cancer: changing treatment strategies and results of surgical intervention. Ann Surg Oncol 2009;16:361-70.

11. Timmerman RD, Bizekis CS, Pass HI, et al. Local surgical, ablative, and radiation treatment of metastases. CA Cancer J Clin 2009;59:145-70.

12. Lan YT, Jiang JK, Chang SC, et al. Improved outcomes of colorectal cancer patients with liver metastases in the era of the multidisciplinary teams. Int J Colorectal Dis 2016;31:403-11.

13. Wanis KN, Pineda-Solis K, Tun-Abraham ME, et al. Management of colorectal cancer with synchronous liver metastases: impact of multidisciplinary case conference review. Hepatobiliary Surg Nutr 2017;6:162-9.

14. Van Cutsem E, Cervantes A, Adam R, et al. ESMO consensus guidelines for the management of patients with metastatic colorectal cancer. Ann Oncol 2016;27:1386-422.

15. Diagnosis And Treatment Guidelines For Colorectal Cancer Working Group CSOCOC. Chinese Society

Cite this article as: $\mathrm{Li} X$, Chen Q, Bi X, Zhao J, Li Z, Zhou J, Huang Z, Zhang Y, Mao R, Zhao H, Cai J. Effects of multidisciplinary team on the outcomes of colorectal cancer patients with liver metastases. Ann Palliat Med 2020;9(5):2741-2748. doi: 10.21037/apm-20-193 of Clinical Oncology (CSCO) diagnosis and treatment guidelines for colorectal cancer 2018 (English version). Chin J Cancer Res 2019;31:117-34.

16. Dindo D, Demartines N, Clavien PA. Classification of surgical complications: a new proposal with evaluation in a cohort of 6336 patients and results of a survey. Ann Surg 2004;240:205-13.

17. Nordlinger B, Sorbye H, Collette L, et al. Survival after peri-operative chemotherapy with folfox 4 and surgery for resectable colorectal cancer liver metastases. Final results of the eortc intergroup randomized phase III study 40983. 9th World Congress on Gastrointestinal Cancer. 2007.

18. Mentha G, Majno P, Terraz S, et al. Treatment strategies for the management of advanced colorectal liver metastases detected synchronously with the primary tumour. Eur J Surg Oncol 2007;33 Suppl 2:S76-83.

19. Adam R, Delvart V, Pascal G, et al. Rescue surgery for unresectable colorectal liver metastases downstaged by chemotherapy: a model to predict long-term survival. Ann Surg 2004;240:644-57; discussion 657-8.

20. Choti MA. Chemotherapy-associated hepatotoxicity: do we need to be concerned? Ann Surg Oncol 2009;16:2391-4.

21. Jung SM, Hong YS, Kim TW, et al. Impact of a Multidisciplinary Team Approach for Managing Advanced and Recurrent Colorectal Cancer. World J Surg 2018;42:2227-33.

22. Davies AR, Deans DA, Penman I, et al. The multidisciplinary team meeting improves staging accuracy and treatment selection for gastro-esophageal cancer. Dis Esophagus 2006;19:496-503.

23. Rummans TA, Clark MM, Sloan JA, et al. Impacting quality of life for patients with advanced cancer with a structured multidisciplinary intervention: a randomized controlled trial. J Clin Oncol 2006;24:635-42.

24. Fennell ML, Das IP, Clauser S, et al. The organization of multidisciplinary care teams: modeling internal and external influences on cancer care quality. J Natl Cancer Inst Monogr 2010;2010:72-80. 